\title{
IMS Compliant Ontological Learner Model for Adaptive E-Learning Environments
}

\author{
https://doi.org/10.3991/ijet.v14i16.10682 \\ Othmane Zine ${ }^{(\bowtie)}$, Aziz Derouich, Abdennebi Talbi \\ Sidi Mohamed Ben Abdallah University, Fez, Morocco \\ othmane.zine@usmba.ac.ma
}

\begin{abstract}
It has been proven that adopting the "one size fits one" approach has better learning outcomes than the "one size fits all" one. A customized learning experience is attainable with the use of learner models, the main source of variability, in adaptive educational hypermedia systems or any intelligent learning environment. While such a model includes a large number of characteristics which can be difficult to incorporate and use, several standards that were developed to overcome these complexities.

In this paper, the proposed work intents to improve learner's model representation to meet the requirements and needs of adaptation. We took IMS-LIP, IMSACCLIP and IMS-RDCEO standards into consideration and incorporated their characteristics to our proposed learner model so that it conforms to international standards. Moreover, the suggested learner model takes advantage of the semantic web technologies that offer a better data organization, indexing and management and ensures the reusability, the interoperability and the extensibility of this model. Furthermore, due to the use of ontologies, the metadata about a learner can be used by a wide range of personalization techniques to provide more accurate customization.
\end{abstract}

Keywords - Elearning; adaptive educational environments; personalization; learner characteristics; learner modeling; ontological engineering

\section{$1 \quad$ Introduction}

E-learning has moved from traditional content delivery approaches to a personalized, adaptive and learner-centered knowledge transfer. While massive open online courses, learning management systems and other standard e-learning platforms follow the "One size fits all" principle, disregarding user needs and preferences and offering the same courses to all students, adaptive hypermedia and other intelligent adaptive learning environments better match the expectations of each learner apart, by identifying his skills, goals and learning methods and adapting the courses and the interfaces to his own level, rhythm, and preferences.

It is claimed that hypermedia systems meet the objective of adaptation and are a suitable and effective option for providing personalized learning paths and appropriate intervention in selecting and displaying each learning object or activity in line with the 
learners' individual differences. This adaptation is essentially based on a meticulous design of the learner model, which is the core component of any adaptive learning system. It incorporates all the learner's pedagogical and psychological characteristics that are necessary for the system to identify the learner (knowledge, learning styles, psychological states, etc.), which guarantees an accurate and proper performance.

Recent developments in the semantic web have captivated researcher on using these technologies for developing adaptive e-learning systems (i.e. learner modeling, domain knowledge representing, etc.). From this perspective, the semantic web allows the provision of knowledge and learning content in various forms that might be distributed over a heterogeneous network but with semantic links to each other. It provides annotation based on the semantics of learning resources, easy restructuring of learning design of the learning system and individual delivery of learning materials, which enhance the interoperability, collaboration, content exchange and reuse [1].

"The Semantic Web is an extension of the current web in which information is given well-defined meaning, better enabling computers and people to work in cooperation." [2]. It allows web content to be read, processed and interpreted by humans and machines accurately [3]. Moreover, it provides a framework based on formal logic for structured, distributed and extensible knowledge. The objective of the semantic web is to enable expressing information from the web in a natural and formal language that can be interpreted by intelligent agents. Which enables them to locate, share and integrate information in an automated manner [4].

Semantic Web building blocks are ontologies. They have become a key enabling technology in several fields and are widely used in domains such as the semantic web, artificial intelligence and, in general, wherever there is a need to structure the concepts of a domain [5]. Ontologies provide a suitable mean for representing knowledge due to their flexibility and extensibility in designing concepts and their relationships. An ontology can be defined as "a formal and explicit specification of a shared conceptualization" [6][7], which means that an ontology should capture and share consensual knowledge and should be defined declaratively, structured and machine interpretable and assessable.

We can find in the literature that using ontologies to model the user profile has already been proposed in various applications like web search [8], personal information management [9], human resource management [10] and healthcare [11]. Several attempts have been made to implement ontological learner models in the adaptive educational systems. [12] present an ontology network-based student model the structuring and representation of a student model called ON-SMMILE. It combines the student model ontology with student independent ontologies and organizes the information obtained from the student model in accordance with standard specification. [13] propose a system to improve knowledge management and representation of associated data based on an ontological learner model that uses the VARK learning model to align learner to proper paths of learning. [14] suggest an ontology model called OntoSakai to represent LMS users' context. [15] present an ontological learner modeling to organize the educational information in Healthcare Human Resource Management in Romania. [16] used fuzzy logic and Ontology techniques to model the student's learning behavior to enhance the system's adaptability. [17] outline an ontology-based student model for 
distance learning students. That can be used as an integral ITS module and can be easily accessed from a web-based application. [18] describe learners' model ontology for creating personalized e-Learning systems based on learner's abilities, learning styles, prior knowledge and preferences. [19] introduce a semantic learner model based on the FOAF ontology to support automation of the process of grouping students and preserve at the same time each learner's personal needs and interests.

\section{Basic Concepts}

The aim of adaptive e-learning environments is to offer, using the data picked in the learner model, more suitable learning tasks and create learning materials adapted to particular student characteristics, with the intention of making him learn better, easier and faster.

\subsection{Learner model}

The learner model is a data structure used to describe, record, track, retrieve and update learner's characteristics which may be relevant for adaptive learning. It is the key item in any adaptive E-Learning system. It aims to provide educational resources in a way that meets the needs and expectations of each learner [20]. More specifically, this model provides the necessary information about each learner to the environment to facilitate the learning process and the acquisition of knowledge, the learning path and interface adaption and suitable feedback and support providing [21].

Unlike the learner profile, which is a collection of personal information about the learner recorded without any description or interpretation, the learner model consists of a higher level of abstraction and modeling of this stored information. Researchers claim that in order to provide customization in any e-learning system, it is crucial to store not only the learner's elementary characteristics (e.g. personal information, abilities, prior and current knowledge, goals) in the learner model, but rather catch, as faithfully as possible, the student's psychological state, preferences and reasoning process [22] [23].

\subsection{Learner model representation}

While adaptation requires knowledge about learners, the learner model contains explicitly modeled assumptions that represent the learner's characteristics which are pertinent to the system. The constituents of a learner model are arranged differently in accordance with the design of the environment. There are several techniques for modeling the learner and refining this model. An overview of some of them is presented in the following.

One widely adopted approach for learner modeling is the social model or Stereotypes. It allows the classification of all distinct learners of an adaptive system in several predefined groups based on shared characteristics. Then the system will adopt the same behavior with all the learners belonging to the same group [24]. 
The most common representation of a learner model is the overlay model [25]. It represents a learner's knowledge as a subset of the domain knowledge that represents individual subjects and concepts. While the differential model [26], a variant of the overlay model, sheds light on the gaps between the concepts covered by learner current knowledge and the concepts that should be mastered at the end of the course. Therefore, for both cases, the system will provide the learner with educational material until it covers the needed concepts to reach a certain learning objective (expert's knowledge). These models are inadequate for modeling advanced systems due to their inability to represent the erroneous knowledge that the learner can acquire. In contrast to overlay and differential models, error, buggy, and perturbation learner models represent incorrect beliefs that learners may hold and incorporate information about possible misconceptions or bugs and take into account all incorrect knowledge of the learner to provide suitable advice to correct his mistakes [27]. Yet those models are more powerful but are much harder to develop.

Other widely used approaches are the probabilistic models: Fuzzy logic [28] and Bayesian networks [29] differ from classical set models as they allow representing uncertainty. They improve the accuracy and efficiency of the process of observation and analysis of action sequences. And since there is no direct interaction between the teacher and the student, the presence of uncertainty in the diagnosis of the learner is increased, so, due to their ability to easily represent human concepts, the integration of one of these in the learner model to anticipate the learner's future behavior and performance, improves the adaptability of the system.

And last but not least, ontologies are becoming the typical approach of knowledge representation and have a lot of benefits in this area [30]. They have been proven to be effective means, in the knowledge management field, for describing data within a specific domain in a semantic way [31].

Unlike traditional data structures that only provide a structure for data instances storage, ontologies can express extremely complex relationships between the concepts they represent. They store content in a machine-readable format so as to be perceptible to the human and the machine, which enhance the parsing capabilities. In one hand, owing to their reasoning and inference abilities, they allow new knowledge extraction. In the other hand, they allow the formal representation of abstract concepts and properties to ensure reusability, extensibility, and interoperability of content over the web.

\subsection{Learner model elicitation}

In order to derive learner model information, adaptive learning systems usually solicit the user directly via forms, quizzes, and menus (static acquisition), as the communication flow between the learner and the system requires direct feedback from the learner. Another way to gather this information is through inferring data based on the user's interactions with the system during the learning sessions (dynamic acquisition). The system can mine information from the actions logs by applying machine learning techniques [32]. There are also systems that use a hybrid approach that combines these two approaches (static initialization and dynamic update). 


\section{Learner's Features Taxonomy}

Numerous researches claimed that an accurate definition the learner's characteristics influences and increases considerably the capability and efficiency of learning activities [33] [34] [35].

In the following, we outline a taxonomy of the potential features and characteristics that can describe a learner based on the investigation of existing learner model structures and the analysis of the needs.

Different adaptive systems store different data about users according to the objective of the adaptation. In our case, the user is a learner, so the system should be able to answer questions such as: What is the name of a learner? What is her/his educational level? What is her/his motivational status? What's her/his learning style? What type of media does she/he use for interaction? Or how well does she/he master a certain topic?

As there is an exhaustive choice of the learners' characteristics that can be incorporated in the student model, the selection of the appropriate ones is required. Consequently, we have to select only the necessary and relevant ones in the context of the Moroccan higher education system. We can cite:

- Personal profile: Deals with basic personal information about the learner such as name, first name, age, email, username and password, affiliation, educational level, and deficiencies.

- Knowledge: Includes learner's background and acquired skills and knowledge level that are specific to a domain. This information can be evaluated via tests and questionnaires during the learning session. Moreover, it includes domain independent knowledge such as computer mastery, mastered languages and other official certificates.

- Errors: Are mistakes that can be defined as non-recurring bad answers, that learners can easily fix by themselves.

- Misconceptions: Refer to the correct execution of an incorrect procedure and erroneous conceptions or mistaken notions that are symptomatic of a faulty line of thought.

- Goals: Learning goals of the learner defined in terms of knowledge and/or skills to acquire either at the end of the course or during the learning session.

- Assessment: Learner's taken tests and evaluations, the obtained scores, the acquired knowledge, and level of mastery [36].

- Preferences: Different preferences regarding the different aspects of the learning environment such as the coloring scheme, the fonts and the size of the text.

- Learning styles: Designate the learning choices and learning differences that affect how a learner collects and deals with the learning objects [37] [38].

- Motivational states: The adaptive learning environment should interpret the motivation level of the learner and adapt its behavior to their state and assign suitable tasks in response to these emotions. Motivation is measured using parameters such as the effort, interest, boredom, distraction, and persistence, etc. [39]. 
- Cognitive abilities: Refer to intellectual skills or the mental process to acquire knowledge such as attention, knowledge, memory, perception, concentration, collaboration skills, decision making, reasoning, and critical thinking.

\section{Current Learner Model Standards Benchmark}

Standardization doesn't address only the learning objects, but also the learner information and so, learner characteristics should be well defined to ease their use in different platforms of e-learning and to grant a more accurate personalization.

Moreover, standards allow reducing variability in data models used to maintain learner profile records. Within this context, researchers in the educational field have deeply investigated those characteristics and attempted to model the learner data in a formal way that promotes reuse and interoperability.

Several standardization institutions such as the IEEE Learning Technology Standards Committee and IMS Global Learning Consortium have developed norms to meet that purpose, we review below the most important and most prominent ones.

\subsection{IEEE PAPI Learner (Public and Private Information for Learners) [40]}

Developed by the IEEE LTSC (Learning Technology Standards Committee), is one of the first proposals of a standard framework for constructing and classifying learner's data. It's a format which specifies the syntax and semantics of learner records and incorporates the Dublin Core metadata element set.

This specification provides a minimal amount of learner information and aims at supporting the representation, retrieval, and interchange of learner models among different educational systems. And it supplies researchers or developers intending to build a learner model with a foundation for the development of learner models and a standardized and growing source of data.

$P A P I$ logically splits the learner information into six distinct and expandable subsets:

- Learner personal: Presents the personal information about the learner such as his name, address and email.

- Learner relations: Describes the relationships with the other users of the platform such as learners and tutors;

- Learner security: Holds the user's security details and access rights such as passwords, public and private keys;

- Learner preference: Indicates information targeted to improve the human-computer interactions and provide the optimum learning experience such as learning styles, preferred language or disabilities;

- Learner performance: Refers to the record of the learner's history and measured performance such as grades, progress and goals that is created and used to offer the most advantageous and appropriate learning path;

- Learner portfolio: Aims at presenting and evidencing learner' achievements and skills by providing a collection of a learner's accomplishments and experience. 


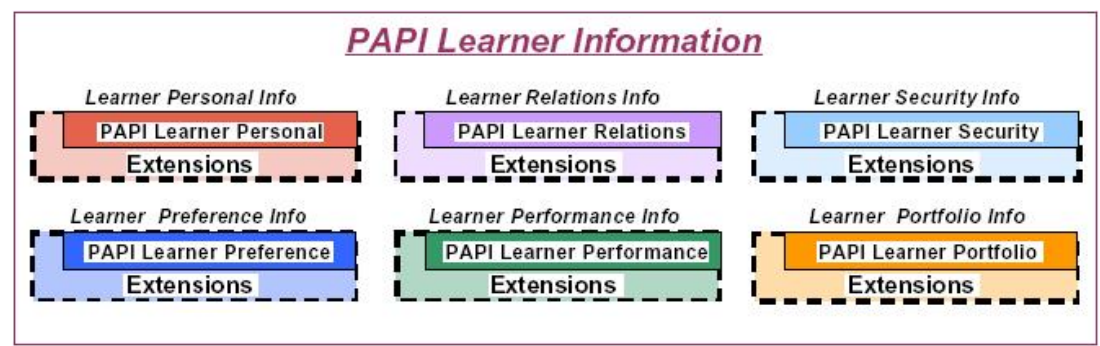

Fig. 1. IEEE PAPI learner information

\subsection{IMS Global Learning Consortium specifications}

The IMS Global Learning Consortium developed various specifications and guidelines relevant to learner modeling and content and interfaces adaptation to meet the needs of individuals such as:

LIP (Learner Information Package): The Learner Information Package (LIP) specification defines an $X M L$ structure that describes the essential characteristics of a learner and comprises information comparable to that covered by a learner's CV for recording and management purposes.

Aiming at exploring learning opportunities for learners, it promotes the interoperability and cooperation between software applications, that use and might need to exchange and share a part of the collection of learner information (both data and metadata), (e.g. learning management systems, knowledge management systems, resume repositories, or any other e-learning environment) by defining a set of packages that can be used to import and export data from an IMS compliant system.

LIP structures the data into eleven segments that represent the primary data structures that are mandatory to support the learner information. These segments are:

- Identification: Describes the personal data on the learner, (name, age, address, email, etc.)

- Goal: Provides information about the purpose of the learning task, the intended career and other objectives such as personal goals and aspiration

- QCL (Qualifications, Certifications \& Licenses): Lists all of the learner's qualifications, certifications and licenses obtained from recognized authorities

- Activity: Contains a description of the learning related activities in any state of completion (training, work experience, etc.)

- Transcript: Presents an institutionally-based summary of academic results and achievements

- Interest: Describes the learner's hobbies and recreational activities

- Competency: Describes the skills, experience and knowledge acquired, etc.;

- Accessibility: Describes general accessibility such as language abilities and preferences, disabilities, eligibility and learning preferences;

- Security key: Holds security data of a person, such as passwords, access rights and security keys assigned to a learner; 
- Affiliation: Represents information records about the professional associations and the organizations where the learner has a membership (work groups);

- Relationships: Describes the relationships between core data elements used to store the learner information used in this model.

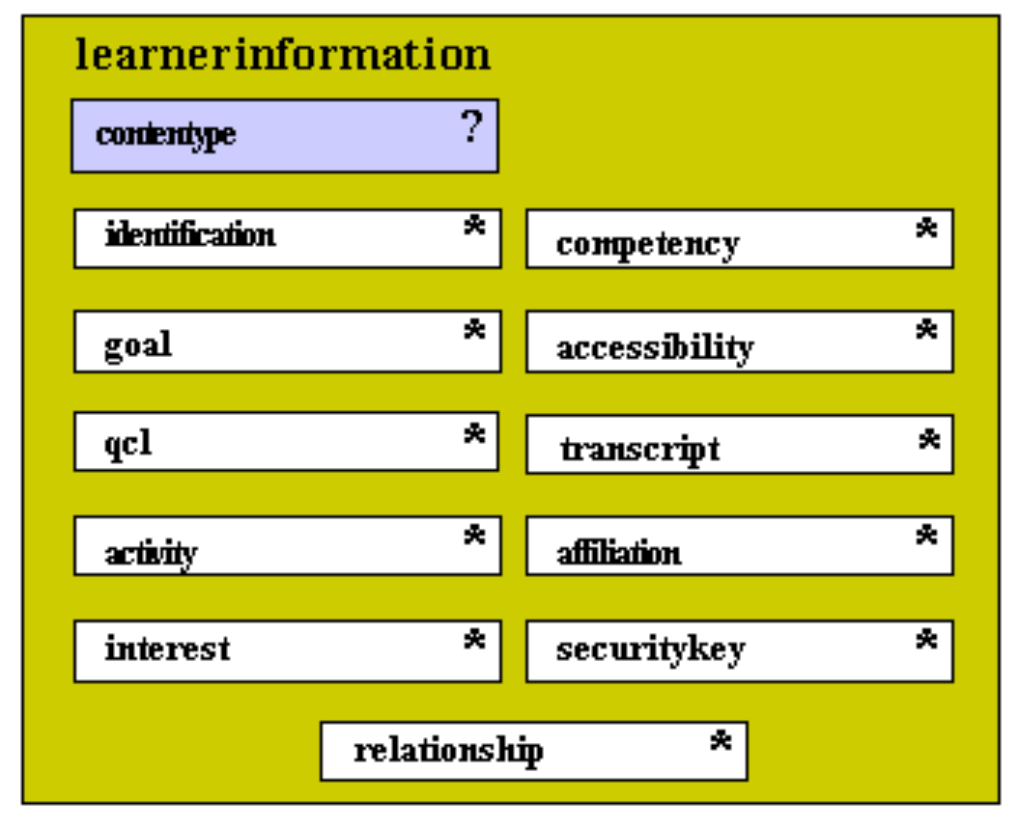

Fig. 2. IMS LIP learner information

ACCLIP (Accessibility for Learning Information Package): IMS ACCLIP is one of the first initiatives of the IMS Accessibility Working Group to extend the LIP specification to address accessibility issues and allow learner accessibility preferences to be defined. It adjusts the $<$ accessibility $>$ element in IMS-LIP, trough discarding the $<$ disability $>$ element and including the $<$ AccessForAll $>$ one.

The ACCLIP specification is about individualization and customization and is not disability-centric, which means it can be used for both the standard system and the assistive ones. And so, it improves accessibility not only for people with disabilities but also for non-disabled learners by assuming that any learner will have different access preferences depending on any number of factors or constraints like low-bandwidth or small screens. It allows the system to adapt the selection of learning content, its display, and controls to match the learner's individual needs and preferences by enabling the learner to specify his accessibility preferences for the manner of displaying the resource, the way of controlling it and the form of the delivered content [45].

Accessibility preferences are sorted into three classes: the ones related to the content, those relevant to the content display and those in touch with the control of the content. Content preferences describe alternative or equivalent types of content that the learner might choose such as the audio descriptions instead of the visual content which can be 
relevant for the visually impaired or text instead of audio content for those that might not have speakers on hand while learning. Display preferences describe how the learner wants to have the interface and content displayed. Control preferences define alternative ways of handling the device and describe how the learner prefers to control it (standard keyboard/virtual keyboards).

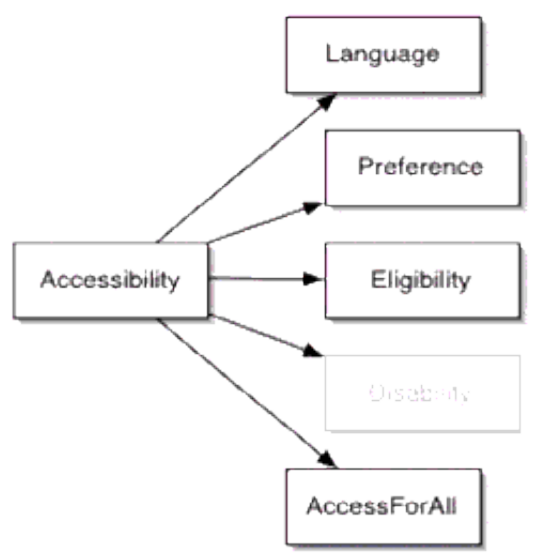

Fig. 3. IMS AccLIP information model

RDCEO (Reusable Definition of Competency or Educational Objective): IMS $R D C E O$ provides a common specification of learner's competencies, using unstructured textual definitions, and disregarding the usage context. It defines a minimalist but extensible information model that can be used to describe, reference and exchange definitions of competencies, mainly in the context of e-learning.

This specification supports the representation of competency main characteristics in a formal way (competency includes skills, knowledge, learning outcomes, etc.). Competencies can be those of a career plan or those of a learning plan (e.g. prerequisites representation or learning outcomes definition) and can be associated to a globally unique reference, which grants interoperability between knowledge management systems (e.g. learning systems, human resource systems, skills repositories, etc.).

However, it does not define how competences would be used as part of a learning process, assessed and certified.

The $R D C E O$ Information Model defines four categories to characterize a competency:

- Identifier: The unique, permanent and sufficient label to reference the competency in any other system

- Title: A short textual description of the competency and is human readable and recognizable

- Definition: A structured and optional description that provides a definition of the competency 
- Description: An optional text-area, interpretable only by a human and which gives a more complete definition of the competency

Identifier and Title are the only mandatory ones.

\subsection{FOAF (Friend of A Friend)}

Founded by Dan BRICKLEY and Libby MILLER in the mid-2000, FOAF is an open source and community-lead project with the goal of linking people and information using the Web. It consists of a Linked Data system expressed using the Resource Description Framework $(R D F)$ and the Ontology Web Language $(O W L)$, in order to define a machine-readable ontology characterizing people, their interests and activities, documents, organizations, and relationships between them. This specification incorporates useful classes and properties for describing people online and can be easily coupled with other vocabularies, which grants the capture of a valuable collection of metadata[17].

FOAF vocabulary is not a standard in the sense of ISO or W3C Process Standardization, but it is managed by following the style of the W3C's standards work ( $X M L$, $R D F$, and $O W L$ ), which makes all $F O A F$ documents well-formed $O W L / R D F / X M L$ documents.

FOAF incorporates five basic categories to represent a profile:

- Person: Includes a basic description of the leaner such as name, age, address, email, etc.;

- Document and image: Holds information about a document or an image related to the learner;

- Organization: Points to the social institutions the learner is a member of;

- Online account: Stores information related to learners' accounts;

- Projects and groups: Store information about the groups or projects the learner participates in.

\subsection{EduPerson}

Defined jointly by INTERNET2 and EDUCAUSE, eduPerson standard is an attribute schema that intends to standardize research and higher educational user and organizational characteristics by providing a practical common list of attributes and definitions for inter-institutional data exchange.

It deals with information similar to the one found in an employee information system (e.g. data about the person and the organization to which he belongs) and incorporates bindings to an LDAP object class designed to facilitate communication between universities, notably to exchange data about people amongst US ones.

And considering that its aims at exchanging data, eduPerson provides very detailed descriptions comparing to other standards and allows only authorized users and services to access information and that is done disregarding the location or the manner of storage of the original information. 
The learner's information which is addressed by this standard is classified in the two categories:

- General attributes, which holds learner's general information about the learner, such as address, name, security settings, and information about the organization the learner belongs to, e.g. name, location, etc.

- Attributes is created to facilitate collaboration and communication between institutions and include learner's affiliation, learner's ID, affiliation, etc.

\subsection{Comparison of the standards}

The table below summarizes the differences between all learner models described above based on their proposed taxonomies and supported features.

Table 1. Comparison between standards for learner information

\begin{tabular}{|c|c|c|c|c|c|c|}
\hline \multirow{3}{*}{$\begin{array}{c}\text { Supported } \\
\text { features/aspects }\end{array}$} & \multicolumn{6}{|c|}{ Reference Model } \\
\hline & \multirow{2}{*}{ PAPI } & \multicolumn{3}{|c|}{ IMS } & \multirow{2}{*}{ FOAF } & \multirow{2}{*}{ eduPerson } \\
\hline & & $L I P$ & ACCLIP & RDCEO & & \\
\hline Personal data & + & + & & - & + & + \\
\hline Competencies & - & $+/-$ & & + & & \\
\hline Affiliation & & + & & & + & + \\
\hline Accessibility & & & + & & & \\
\hline Info portability & + & + & & & + & + \\
\hline Personalization & + & + & & & + & \\
\hline Recording Achievements & + & + & & & & \\
\hline Relations and Community building & $+/-$ & & & & + & $+/-$ \\
\hline Learning Styles & + & + & & & & \\
\hline Academic performance & + & + & & - & & \\
\hline Preference & $+/-$ & $+/-$ & & - & & \\
\hline Security & + & + & & & $\mathrm{x}$ & + \\
\hline Goal & & + & & & & \\
\hline Disability & & + & + & & $\mathrm{x}$ & $+/-$ \\
\hline Certification & + & + & & & & \\
\hline Portfolio & + & + & & & & \\
\hline Learning objective & + & - & & - & & \\
\hline
\end{tabular}

The presentation of the main characteristics of the aforementioned standards confirmed the common belief which states that PAPI and IMS-LIP are the most used and important ones due to the completeness of the plethora of characteristics they offer and features they support. Nevertheless, both standards have some shortcomings. PAPI categories do not allow a detailed description of all the previously stated learner information. While $P A P I$ is a standard that considers the performance information as the most important information about a learner, it neither takes into account learning data (e.g. learning activities) nor covers the goal and competencies categories that can be used for recommendation and filtering techniques. IMS-LIP was able to overcome PAPI imperfections and allows online learning systems to be better adapted to the needs of the learner by proposing a better categorization and adopting a CV alike description. 
Although relations to other people don't figure explicitly in IMS-LIP, they can be represented by relationships between different records using the identification category.

The other IMS specifications (e.g. IMS-ACCLIP and IMS-RDCEO) were developed to serve specific purposes (resp. accessibility and competencies) and propose a better representation of other information that was not raised by IMS-LIP.

EduPerson is the most detailed and suitable for collecting data and transferring it between institutions, but it's only used to point to documents. FOAF is the only model that explicitly outlines learner's relations with others and points directly to other learner profiles. But none of them hold any description of performance or preferences which shows that they were not developed to support personalization.

Some of these standards share a set of common learner characteristics. It is a usual practice to produce a learner model combining different learner standards and profiting from their unique benefits and overcome their shortcomings.

\section{$5 \quad$ Learner Model Ontology}

Ontologies construction is expected to incorporate methods and techniques used in software engineering. In the development process of our ontology, we followed the [49] method that consists of seven phases as shown in the figure below. These phases aren't strictly sequential but follow an iterative process.

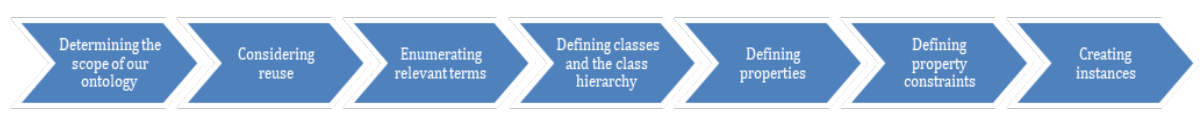

Fig. 4. Development process of the proposal

\subsection{Development process}

Determining the scope of our ontology: In this step, we tried to define the purpose and the coverage of our ontology as It's very important to define from the beginning what the ontology is going to answer. And we aim to have a simplistic representation and avoid to make the schema overcomplex and unusable and hard to maintain.

We have defined the questions for which the information included in our ontology should provide answers (competency questions) and we used this set of questions as templates we keep in mind before starting the ontology engineering steps.

Table 2. Excerpt of competency questions

\begin{tabular}{|l|l|}
\hline Q1 & Which learner's characteristics should be considered when addressing a learner? \\
\hline Q2 & What's the knowledge level of leaner A in domain B? \\
\hline Q3 & What are the cognitive abilities of learner C? \\
\hline
\end{tabular}

Considering reuse: We investigated the learner modeling standards mentioned in section 5 as well as upper, domain-specific, reference ontologies and ontologies that have been validated through use in other applications. We've taken into consideration reuse 
in the ontology development in order to save effort and ensure that there will be interoperability between our ontology and other ontologies since that our ontology might have to interact with systems that use other ones. And so, terms that we defined in our ontology can be reused, for example, in job seeking system to define someone's competencies.

Enumerating relevant terms: Basically, we started by enumerating all the important terms in the learner modeling field that we'll use to build our ontology. We went through articles and standards to dig specific terms, their properties and constraints on these properties. These terms are a starting point to create classes of our ontology.

Table 3. Some of the listed terms

\begin{tabular}{|l|l|l|}
\hline \multicolumn{1}{|c|}{ Learner } & \multicolumn{1}{c|}{ Learner style } & \multicolumn{1}{c|}{ Disability } \\
\hline Novice & Reflexive & Motivation \\
\hline Competency & Name & Certificate \\
\hline
\end{tabular}

Defining classes and the class hierarchy: We categorized elements with similar properties to create classes and define the class inheritance. We used a combination of the top-down and bottom-up modes of development: We started with the most pertinent concepts, then specialized the most general ones and organized the specific information that we collected about the individuals in more general classes to create a taxonomic hierarchy of our classes.

Defining properties: We defined attributes of instances of each class and their relation to other instances (slots) as well as the relationships that link the classes of characteristics of each class.

To provide the relationships amongst two individuals from given classes, we specify the mutual OWL object properties that are in multiple forms (e.g. has Affective] State and its inverse property is Affective State Of) and we specified datatype properties that are used to link objects to datatypes (e.g. has Birth Date, has Name).

Defining property constraints: After defining classes and properties on these classes we defined constraints on these properties. Constraints are used to limit the set of possible values for a property.

We determined the domain and range of each property as well as its cardinality, value type, minimum, maximum, and default values.

Creating instances: We created instances of classes as well as the corresponding slot values in order to specialize classes and populate specific individuals.

\subsection{Our proposal}

The figures below depict the graphical representation of the developed learner ontology, which is a detailed version of the concept hierarchy. It represents a well-structured and shared vocabulary that tends to capture all the concepts presented in section 3 for describing learner profiles and aims at answering queries about learners' static and dynamic characteristics. Our ontology is compliant with the IMS standards (LIP, $A C C L I P, R D C E O)$. 
We decided, in our modeling approach, to arrange learner model characteristics into facets. The Learner class is the key concept of our hierarchy as it includes all specific details regarding learners. It's associated with the corresponding sub-classes through has Profile, has Education and has Personality, object properties.

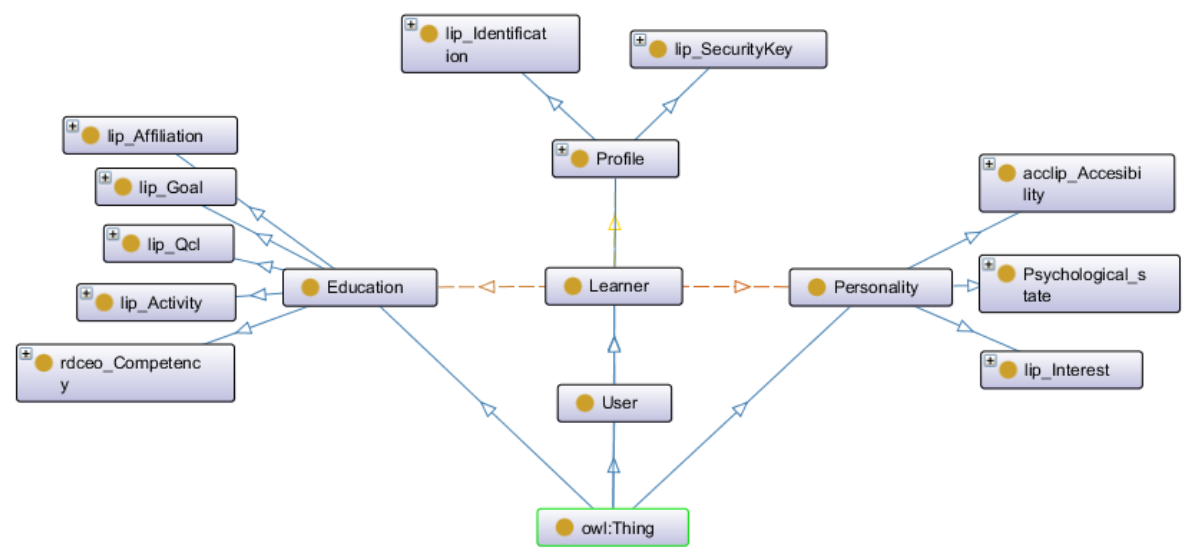

Fig. 5. Learner model ontology

Profile: Is composed of the Identification class (figure 6) and the SecurityKey one. It represents each user's individual static information that will persist and won't evolve during sessions such as user's name, gender, email, etc. which allows the system to identify and address every user. It contains security data of users too, in our case it holds passwords.

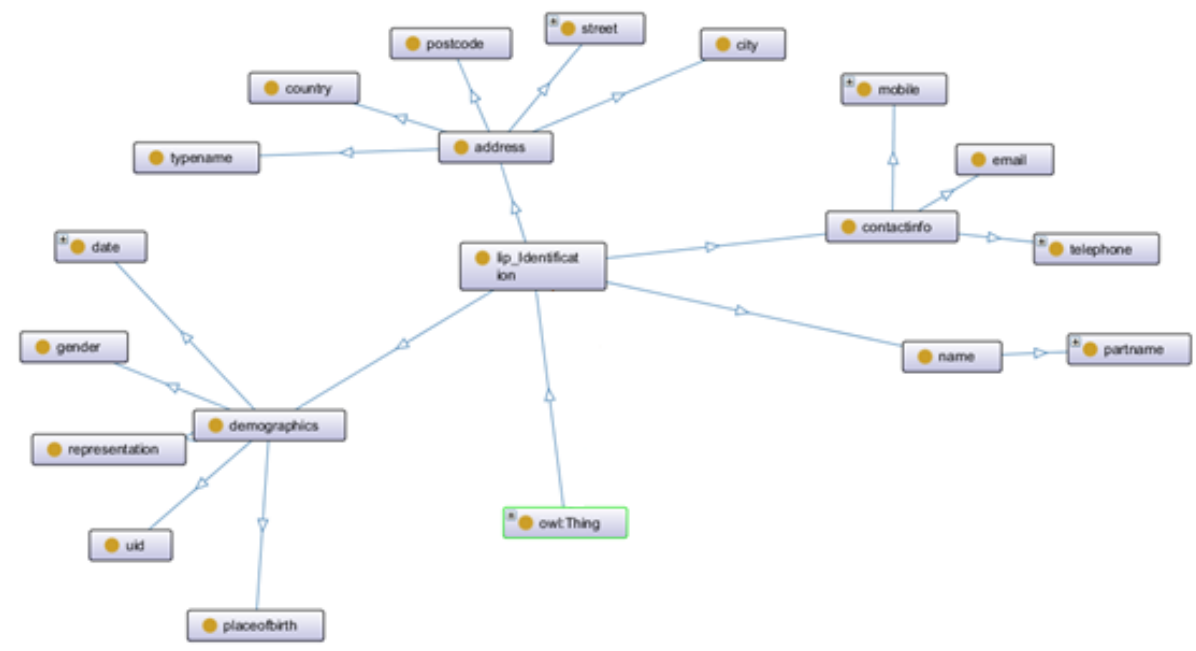

Fig. 6. Identification class 
Education: Contain asserted and inferred data about learner's education and is composed of four sub-classes:

- The Affiliation class represents data about the associations and organizations where the learner has a membership (workgroups) such as information about the organization, the membership number and the undertaken role of the learner.

- The $Q C L$ class is based on the learner's previous education and experience and lists all of the learner's qualifications, certifications, and licenses obtained from recognized authorities. These last have a specific registration number and might have a validation period.

- The Goal class provides information about the personal aspiration, the expected job or career, the aim of the undertaken learning tasks and other objectives.

- The Activity class contains a description of the learning activities and tasks such as a description of the activity, the state of completion and evaluation details.

- The Competency class (figure 7) was created according to the IMS-RDCEO standard and contains descriptions and references of competences. It provides a flexible schema for describing, expressing and exchanging subtle details of competencies, offers different means to assess diverse learner's aspects such as skills, knowledge, abilities, outcomes, and objectives described in learning or professional fields. Each competence might have a quantitative and/or qualitative assessment.

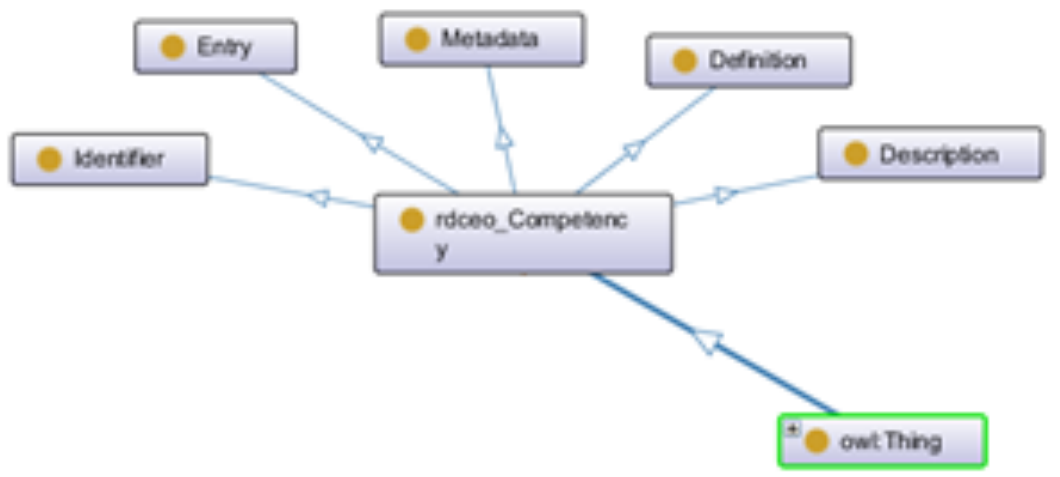

Fig. 7. Competency class

Personality: Represents learner's accessibility preferences as well as his psychological state and interests.

- The Accessibility class (figure 8) deals with accessibility issues regarding language, eligibility and learning cognitive preferences concerning material display, system control, and the desired content transformations or enhancements.

- The Interest class holds information about learner's hobbies or recreational activities. 


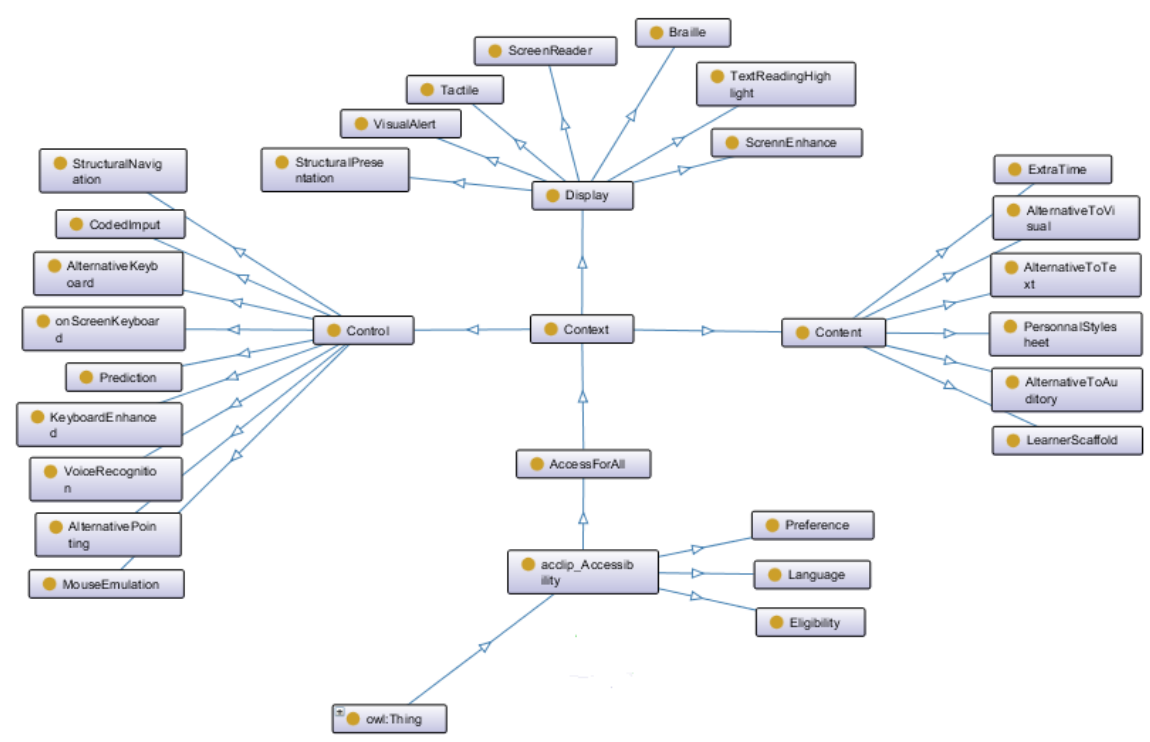

Fig. 8. : Accessibility class

- The Psychological State class (figure 9) holds relevant information about learner's affective states, learning styles and cognitive and metacognitive factors. The system will observe how a learner reacts to these different types of stimuli and use this information to select suitable instructional content and strategies and provide a tailored learning experience. This class, its respective sub-classes and their way of representation have been the subject of a profound study and will be detailed in another paper.

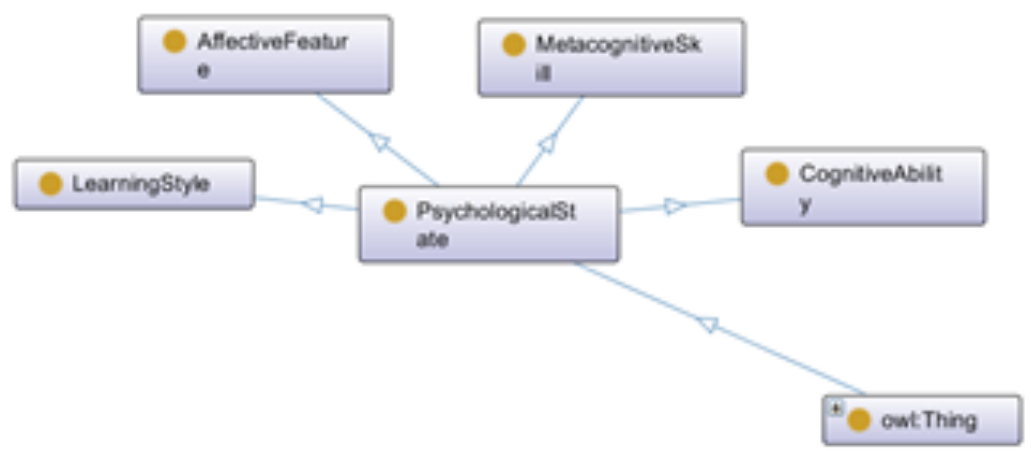

Fig. 9. Psychological State class 


\subsection{Scenario}

The figure below depicts the use scenario. This scenario describes the actions and reactions between the system and the learner.

When a first-time user accesses the platform, he is asked to register and fill a form about the personal information (name, demographics, contact info), password, qualifications, and interests. Then he is invited to respond to a set of psychologically-oriented questions in order to determine his psychological features such as his learning style and cognitive abilities and adjust his preferences stings via a menu of options to customize the presentation.

Once done, and whether the learner has just completed registration or already has an account and has just logged in, he is requested to define his learning goal and sets for placement pretest to evaluate his knowledge on the field before accessing the course. The system initializes the learner's knowledge about this field and assigns him a level according to the result of this pretest.

Relying on the previously cited collected information, the system goes on gathering the appropriate course via selecting and combining relevant learning concepts as well as presenting them in a customized way to build the learning activity.

Hence, the learner starts the adapted learning activity that might contain sub-activities and evaluation tasks. Finally, when the course learning activities are completed and all the sub-goals are achieved, the goal is reached and the competency is mastered.

During learning sessions, the system infers data from learner interactions and assessments and updates learner's information. 


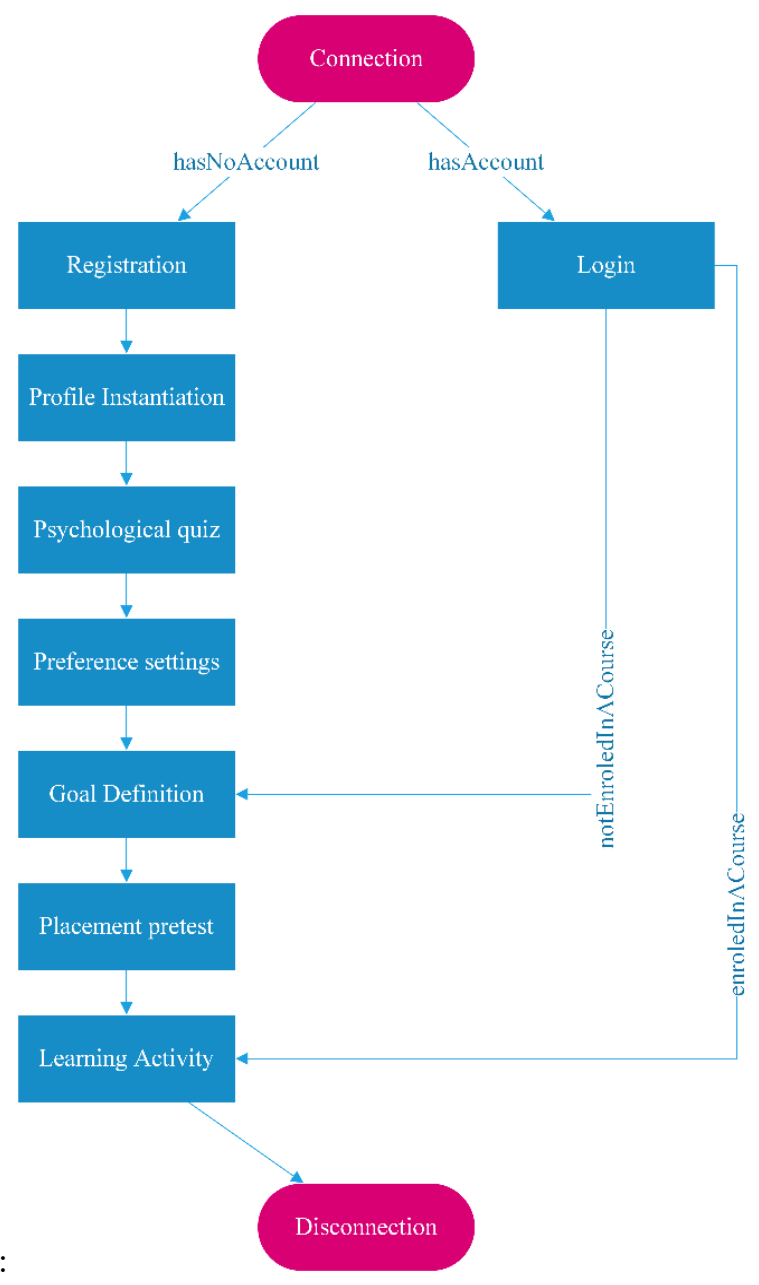

Fig. 10.Scenario

\subsection{Evaluation and validation of the proposed ontology}

We used the reasoner Hermit 1.3.8.413 to evaluate and verify our ontology. A reasoner is a tool used to infer information that is not explicitly contained within the ontology and interpret the semantics of the objects included in an ontology model and to extract information from it. It allows consistency, subsumption, equivalence, instantiation checking of the proposed ontology. The reasoning may be done at different levels. While from the learner's answer to a question, the learner's correct or buggy knowledge can be inferred, from learner's result in assessment, the system can obtain the acquired competency and the degree of mastery and from the assessment's type learner's abilities might be deduced (memory, learning speed). 
Hermit is an $O W L-D L$ reasoner that offers a set of functionalities to inspect $O W L$ documents such as identifying conflicting axioms by mean of the consistency function and grants datatypes verification, model evaluation, anomalies identification, and correction.

The evaluation verifies the syntax and semantics of the refined ontology by considering the scenario and the end users so to have the learner model apt to be incorporated in the adaptive e-learning system.

The result of the reasoner indicates that there is no contradiction between axioms. Which means that the implemented model is in accordance with $O W L 2 Q L$ specifications which are a good compromise between computational weight and expressiveness.

\section{Conclusion and Future Work}

In this paper, we present an ontology-based approach to model learners enrolled in distance learning. We started from a detailed statement and collection of the academic learner's characteristics which are considered relevant for adaptation and reviewed the main modeling approaches available in the literature. Then, we studied the current learner modeling standards in educational systems. After that, we described the development process, the scenario and the validation of our proposal.

One of the key benefits of this approach is the integration of semantic rules which once combined with inference mechanisms provide additional knowledge about the learners. The most challenging part of our research has been the selection of the most appropriate characteristics to be included in the ontology that would be, at the same time, compliant with the current learner modeling standards (the IMS standard in our case).

We choose to use ontologies because of their knowledge representation, reuse, sharing and modeling abilities. In an e-learning context, ontologies allow the semantic annotation of data (e.g. learner profiles, educational content) which offers a better data organization, indexing, and management in order to deliver to the learner relevant educational materials according to her/his ontology-based profile. Also, the use of interoperable representation of learner models allows adaptive e-learning systems to build, maintain and update their learner models with data from all of the different systems that the learner uses.

We can exploit the semantics contained in the metadata of the learner model and apply semantic indexing and clustering to group learners that share similar characteristics. This would lead to more accurate resources and learning activities recommendation to learners belonging to the same group and help us to deal with the cold start limitations.

We can take advantage of these semantics to create complemental and supportive pairs or groups when dealing with collaborative learning activities (e.g. having at least a person with a competency that is indispensable to the completion of a project or requesting automatically learners that pursue the same learning goal to join the same forums and discussions.). 
In the development of our proposal, we defined several questions that it should be able to answer. Then, we categorized the characteristics and organized them into a hierarchy. We realized the learner model ontology by means of the ontology editor Protégé 5.2.0 We validated it by means of the reasoner Hermit 1.3.8.413 to demonstrate its completeness, expressiveness, and consistency.

On the way of constructing and building an ontological adaptive hypermedia, we plan in the next work to dig and detail all the aspects the "psychological state" facet that deals with learning styles, affective features, cognitive abilities, and metacognitive skills in order to enrich our ontology. The next steps would be the implementation of an integral module allowing the creation, search and update of the profiles via direct communication with the learner model ontology and the modeling of another ontology describing learning objects (domain model).

\section{$7 \quad$ References}

[1] N. Capuano and M. Gaeta, "An intelligent Web teacher system for learning personalization and semantic web compatibility," in Eleventh International PEG Conference, 2003.

[2] T. BERNERS-LEE, J. HENDLER, O. LASSILA, and Al., "The semantic web," Sci. Am., vol. 284, pp. 28-37, 2001. https://doi.org/10.1038/scientificamerican0501-34

[3] D. Dicheva, Handbook on Information Technologies for Education and Training. Berlin, Heidelberg: Springer Berlin Heidelberg, 2008.

[4] S. Grimm, P. Hitzler, and A. Abecker, "Knowledge representation and ontologies," Semant. Web Serv. Concepts, Technol. Appl., pp. 51-105, 2007.

[5] S. Mellouli, F. Bouslama, and A. Akande, "An ontology for representing financial headline news," Web Semant. Sci. Serv. Agents World Wide Web, vol. 8, no. 2-3, pp. 203-208, Jul. 2010. https://doi.org/10.1016/j.websem.2010.02.001

[6] T. R. Gruber, "Toward principles for the design of ontologies used for knowledge sharing?," Int. J. Hum. Comput. Stud., vol. 43, no. 5-6, pp. 907-928, 1995.

[7] N. Guarino, D. Oberle, and S. Staab, "What Is an Ontology?," in Handbook on Ontologies, Berlin, Heidelberg: Springer Berlin Heidelberg, 2009, pp. 1-17. https://doi.org/10.1007/978-3-540-92673-3 0

[8] S. Lawrence, "Context in web search," in IEEE Data Engineering Bulletin, 2000, pp. 2532.

[9] V. Katifori, A. Poggi, M. Scannapieco, T. Catarci, and Y. Ioannidis, "OntoPIM: How to rely on a personal ontology for personal information management," CEUR Workshop Proc., vol. 175 , no. i, pp. 2-6, 2005.

[10] D. Arena et al., "Towards a Semantically-Enriched Framework for Human Resource Management," in Advances in Production Management Systems. The Path to Intelligent, Collaborative and Sustainable Manufacturing, 2017, pp. 306-313. https://doi.org/10.1007/978-3-319-66923-6 36

[11] F. Ongenae et al., "A probabilistic ontology-based platform for self-learning context-aware healthcare applications," Expert Syst. Appl., vol. 40, no. 18, pp. 7629-7646, 2013. https://doi.org/10.1016/j.eswa.2013.07.038

[12] H. Yago, J. Clemente, D. Rodriguez, and P. Fernandez-de-Cordoba, "ON-SMMILE: Ontology Network-based Student Model for MultIple Learning Environments," Data Knowl. Eng., 2018. https://doi.org/10.1016/j.datak.2018.02.002 
[13] M. Rani, K. V. Srivastava, and O. P. Vyas, "An ontological learning management system," Comput. Appl. Eng. Educ., vol. 24, no. 5, pp. 706-722, 2016. https://doi.org/10.1 $\underline{002 / \text { cae } 21742}$

[14] A. Muñoz, J. Lasheras, A. Capel, M. Cantabella, and A. Caballero, "OntoSakai: On the optimization of a Learning Management System using semantics and user profiling," Expert Syst. Appl., vol. 42, no. 15-16, pp. 5995-6007, 2015. https://doi.org/10.101 6/j.eswa.2015.04.019

[15] L. Bajenaru and I. Smeureanu, "An ontology based approach for modeling e-learning in healthcare human resource management," Econ. Comput. Econ. Cybern. Stud. Res., vol. 49, no. 1, pp. 1-17, 2015.

[16] S. Sani and T. N. M. Aris, "Proposal for Ontology Based Approach to Fuzzy Student Model Design," 2014 5th Int. Conf. Intell. Syst. Model. Simul., no. January, pp. 35-37, 2014. https://doi.org/10.1109/isms.2014.14

[17] I. Panagiotopoulos, A. Kalou, C. Pierrakeas, and A. Kameas, "An ontology-based model for student representation in intelligent tutoring systems for distance learning," IFIP Adv. Inf. Commun. Technol., vol. 381 AICT, no. PART 1, pp. 296-305, 2012. https://doi.org/10.1007/978-3-642-33409-2_31

[18] M. Yarandi, A. R. H. Tawil, H. Jahankhani, and S. A. Hosseini, "Ontology-based learner modelling for supporting personalised e-Learning," 2012 Int. Conf. Interact. Mob. Comput. Aided Learn. IMCL 2012, no. Icc, pp. 113-118, 2012. https://doi.org/10.1109/i $\underline{\text { mcl.2012.6396461 }}$

[19] A. Ounnas, I. Liccardi, H. C. Davis, D. E. Millard, and S. A. White, "Towards a Semantic Modeling of Learners for Social Networks," Hum. Resour. Plan., vol. 5/12/, pp. 102-108, 2006.

[20] F. Hlioui, N. Alioui, and F. Gargouri, "A system for composition and adaptation of educational resources based on learner profile," 2015 5th Int. Conf. Inf. Commun. Technol. Access. ICTA 2015, pp. 1-6, 2016. https://doi.org/10.1109/icta.2015.7426923

[21] C. Buche, R. Querrec, P. Chevaillier, and G. Kermarrec, "Apports des systèmes tutoriaux intelligents et de la réalité virtuelle à l'apprentissage de compétences.," Cogn. - Cah. Rom. Sci. Cogn., vol. 2, no. 2, pp. 51-83, 2006.

[22] S. Gauch, M. Speretta, A. Chandramouli, and A. Micarelli, "User Profiles for Personalized Information Access," in The Adaptive Web SE - 2, vol. 4321, P. Brusilovsky, A. Kobsa, and W. Nejdl, Eds. Berlin, Heidelberg: Springer Berlin Heidelberg, 2007, pp. 54-89. https://doi.org/10.1007/978-3-540-72079-9_2

[23] M. C. Murray and J. Pérez, "Informing and performing: A study comparing adaptive learning to traditional learning," Informing Sci., vol. 18, no. 1, pp. 111-125, 2015. https://doi.org/10.28945/2165

[24] J. Kay, "Stereotypes, Student Models and Scrutability," 2000, pp. 19-30.

[25] M. Aitdaoud, "Standardized modeling learners to enhance the learning service in the ILE Proceedings on Humanities," no. June, 2017.

[26] O. Zine, A. Derouich, and A. Talbi, "Hypermedia multi-agent modeling: a proposition of a learner model agent based on ontologies," in 2nd edition of the International Conference on Pedagogical Approaches \& E-Learning, 2016.

[27] M. Anouar Tadlaoui, S. Aammou, M. Khaldi, and R. Novaes Carvalho, "Learner Modeling in Adaptive Educational Systems: A Comparative Study," Int. J. Mod. Educ. Comput. Sci., vol. 8, no. 3, pp. 1-10, 2016. https://doi.org/10.5815/ijmecs.2016.03.01

[28] K. Chrysafiadi and M. Virvou, "Evaluating the integration of fuzzy logic into the student model of a web-based learning environment," Expert Syst. Appl., vol. 39, no. 18, pp. 1312713134, Dec. 2012. https://doi.org/10.1016/j.eswa.2012.05.089 
[29] E. Millán, T. Loboda, and J. L. Pérez-de-la-Cruz, "Bayesian networks for student model engineering," Comput. Educ., vol. 55, no. 4, pp. 1663-1683, Dec. 2010. https://doi.org/10.1016/j.compedu.2010.07.010

[30] M. Al-Yahya, R. George, and A. Alfaries, "Ontologies in E-Learning: Review of the literature,” Int. J. Softw. Eng. its Appl., vol. 9, no. 2, pp. 67-84, 2015.

[31] C. Snae and M. Brückner, "Ontology-Driven E-Learning System Based on Roles and Activities for Thai Learning Environment," Interdiscip. J. e-Skills Lifelong Learn., vol. 3, pp. 001-017, 2007. https://doi.org/10.28945/382

[32] K. Almohammadi, H. Hagras, D. Alghazzawi, and G. Aldabbagh, “A survey of artificial intelligence techniques employed for adaptive educational systems within e-learning platforms," J. Artif. Intell. Soft Comput. Res., vol. 7, no. 1, pp. 47-64, 2017. https://doi.org/10.1515/jaiscr-2017-0004

[33] H. M. Truong, "Integrating learning styles and adaptive e-learning system: Current developments, problems and opportunities," Comput. Human Behav., vol. 55, pp. 11851193, 2016. https://doi.org/10.1016/j.chb.2015.02.014

[34] O. C. Santos and J. G. Boticario, "Practical guidelines for designing and evaluating educationally oriented recommendations," Comput. Educ., vol. 81, pp. 354-374, 2015. https://doi.org/10.1016/j.compedu.2014.10.008

[35] E. Kurilovas, A. Juskeviciene, S. Kubilinskiene, and S. Serikoviene, "Several semantic web approaches to improving the adaptation quality of virtual learning environments," $J$. Univers. Comput. Sci., vol. 20, no. 10, pp. 1418-1432, 2014.

[36] D. Baneres, X. Baró, A. E. Guerrero-Roldán, and M. E. Rodríguez, "Adaptive e-assessment system: A general approach,” Int. J. Emerg. Technol. Learn., vol. 11, no. 7, pp. 16-23, 2016. https://doi.org/10.3991/ijet.v11i07.5888

[37] Ö. Özyurt and H. Özyurt, "Learning style based individualized adaptive e-learning environments: Content analysis of the articles published from 2005 to 2014," Comput. Human Behav., vol. 52, pp. 349-358, Nov. 2015. https://doi.org/10.1016/j.chb.2015.06.020

[38] M. S. Hasibuan, L. E. Nugroho, P. I. Santosa, and S. S. Kusumawardani, "A Proposed Model for Detecting Learning Styles Based on Agent Learning," Int. J. Emerg. Technol. Learn., vol. 11, no. 10, p. 65, Oct. 2016. https://doi.org/10.3991/ijet.v11i10.5781

[39] S. R. Harandi, "Effects of e-learning on Students' Motivation," Procedia - Soc. Behav. Sci., vol. 181, pp. 423-430, 2015.

[40] I. LTSC, "IEEE P1484.2.1/D8, PAPI Learner - Core Features," Ieee Ltsc, pp. 1-3, 2002.

[41] V. F. Specification and G. Collier, "IMS Learner Information Packaging Information Model," ReVision, no. March, pp. 1-70, 2001.

[42] C. Smythe, F. Tansey, and R. Robson, "IMS Learner Information Packaging Best Practice \& Implementation Guide Final Specification, Version 1.0," no. March, 2001.

[43] V. F. Specification, "IMS Learner Information Package Accessibility for LIP Access for All Use Cases," Learning, no. June, pp. 1-15, 2003.

[44] IMS Global Learning Consortium, "IMS Learner Information Package Accessibility for LIP Best Practice and Implementation Guide." [Online]. Available: https://www.imsglobal.org/accessibility/acclipv1p0/imsacclip_bestv1p0.html. [Accessed: 01-Jan-2017].

[45] L. Harrison and J. Treviranus, "Accessible E-Learning - Demystifying IMS Specifications," in E-Learn: World Conference on E-Learning in Corporate, Government, Healthcare, and Higher Education, 2003. 
[46] IMS Global Learning Consortium, "IMS Reusable Definition of Competency or Educational Objective - Information Model. Version 1.0 Final Specification,” 2002. [Online]. Available: http://www.imsglobal.org/competencies/rdceov1p0/imsrdceo_infovlp0.html.

[47] D. Brickley and L. Miller, "FOAF Vocabulary Specification 0.99," Paddington Edition, 2014. [Online]. Available: http://xmlns.com/foaf/spec/.

[48] D. W. Group, "EduPerson Specification (200312) EduPerson Object Class Specification (200312) Status of this document," pp. 1-34, 2003. https://doi.org/10.26869/ti.9.3

[49] N. F. Noy and D. L. McGuinness, "Ontology Development 101: A Guide to Creating Your First Ontology,” Stanford Knowl. Syst. Lab., p. 25, 2001.

\section{Authors}

Othmane Zine (corresponding author) is currently a Professor in the Department of Electrical Engineering and Computer Science at the Higher School of Technology, Sidi Mohamed Ben Abdellah University, Fez, Morocco.

Aziz Derouich is currently a Professor in the Department of Electrical Engineering and Computer Science at the Higher School of Technology, Sidi Mohamed Ben Abdellah University, Fez, Morocco.

Abdennebi Talbi is currently a Professor in the Department of Mechanical and Industrial Engineering and Industrial Maintenance at the Higher School of Technology, Sidi Mohamed Ben Abdellah University, Fez, Morocco.

Article submitted 2019-04-17. Resubmitted 2019-07-08. Final acceptance 2019-07-08. Final version published as submitted by the authors. 